BULL. AUSTRAL. MATH. SOC.

$41 \mathrm{~A} 10$

VOI. $33(1986), 49-57$.

\title{
ON BOUNDS FOR THE LIPSCHITZ CONSTANT OF \\ THE REMAINDER IN POLYNOMIAL APPROXIMATION
}

\section{David ELLiotT}

Suppose $f$ is a function possessing a $k$ th order derivative, the derivative being Lipschitz continuous of order $\alpha, 0<\alpha \leq 1$, on $[-1,1]$. Let $p_{n}$ be a polynomial of degree $\leq n$ approximating to $f$ on $[-1,1]$ such that if $r_{n}=f-p_{n}$ then $\left\|x_{n}\right\|_{\infty} \leq A n^{-k-\alpha}$. Define

$$
M_{n}(\beta)=\sup _{x_{1}, x_{2} \epsilon[-1,1]}\left|r_{n}\left(x_{2}\right)-r_{n}\left(x_{1}\right)\right| /\left|x_{2}-x_{1}\right|^{\beta},
$$

where $O<B \leq 1$. Upper bounds are obtained for $M_{n}(B)$ when $k \geq 1$ thereby generalizing results previously given for functions which are only Lipschitz continuous on $[-1,1]$.

\section{Introduction}

The recent interest in quadrature rules for the evaluation of Cauchy principal value integrals over finite intervals has required a knowledge of the behaviour of the remainder when a function is approximated by a polynomial. A useful result was a lemma given in 1957 by Kalandiya [2]. More recently, Ioakimidis [1] has given what he calls an "improvement" of Kalandiya's lemma. We shall now summarise these

Received 16 April 1985.

$\$ A 2.00+0.00$.

Copyright Clearance Centre, Inc. Serial-fee code: 0004-9727/86 
results.

Suppose that a given function $f$ is Lipschitz continuous of order $\alpha$, where $0<\alpha \leq 1$, on the interval $[-1,1]$. That is, for every pair of points $x_{1}, x_{2} \in[-1,1]$ we have

$$
\left|f\left(x_{2}\right)-f\left(x_{1}\right)\right| \leq L\left|x_{2}-x_{1}\right|^{\alpha},
$$

where $L$ and $\alpha$ are independent of $x_{1}$ and $x_{2}$. We write $f \in \operatorname{Lip} \alpha$. The Lipschitz constant is the smallest number $L$ for which (1.1) is satisfied and if we know this we sometimes write $f \in \mathrm{Lip}_{L}{ }^{\alpha}$. For each positive integer $n$, let $p_{n}$ be a polynomial of degree $\leq n$ which approximates to $f$ on $[-1,1]$ in some way, and denote by ${ }_{n} n$ the remainder in this approximation so that

$$
r_{n}=f-p_{n} \text {. }
$$

Obviously for $f \in \operatorname{Lip} \alpha, r_{n} \in \operatorname{Lip} \alpha$. Furthermore, we know that $r_{n} \in \operatorname{Lip} \gamma$ for all $\gamma \leq \alpha$. We shall be interested in obtaining bounds for the quantity $M_{n}(\beta)$, say, defined by

$$
M_{n}(\beta)=\sup _{x_{1}, x_{2} \in[-1,1]} \frac{\left|r_{n}\left(x_{2}\right)-r_{n}\left(x_{1}\right)\right|}{\left|x_{2}-x_{1}\right|^{\beta}},
$$

where $B$ is a number whose value may be limited by $\alpha$ (see Theorem 1.1, below). Thus $M_{n}(B)$ is the Lipschitz constant for the function $r_{n}$ considered as an element of the space Lip $\beta$.

The uniform norm for any continuous function $g$ defined on $[-1,1]$ will be denoted and defined in the usual way by

$$
\|g\|_{\infty}=\max _{-1 \leq x \leq 1}|g(x)| \text {. }
$$

It is well known, see for example Meinardus [3, Theorem 43], that if $p_{n}$ is the polynomial of best uniform approximation of degree $\leq n$ to $f \in$ Lip $\alpha$, then $\left\|r_{n}\right\|_{\infty} \leq A n^{-\alpha}$. The results of Kalandiya and Ioakimidis can be given together in the following theorem. (Throughout, $A_{1}, A_{2}, A_{3}, \ldots$ will denote positive constants independent of $n$ ). 
THEOREM 1.1. Stqpose $f \in$ Lip a and $p_{n}$ is a polynomial of degree $\leq n$ such that

$$
\text { (1.5) } \quad\left\|r_{n}\right\|_{\infty} \leq A_{1} n^{-\alpha} \text {. }
$$

Then

(i) for every polynomial of degree $\leq n$ satisfying (1.5) and for $O<B \leq \alpha / 2$,

$$
\text { (1.6) } \quad M_{n}(B) \leq A_{2} n^{-\alpha+2 \beta} ;
$$

(ii) there exists a polynomial of degree $\leq n$ satisfying (1.5) and such that for $0<\beta \leq \alpha$,

$$
M_{n}(\beta) \leq A_{3} n^{-\alpha+\beta} \text {. }
$$

Theorem 1.1 (i) was first stated and proved by Kalandiya [2]. Theorem 1.1 (ii) was stated and proved recently by Ioakimidis [1]. The latter proof makes use of a result due to steckin [4] which we shall state as Theorem 1.2. As a result of it, Ioakimidis' proof is considerably simpler than that of Kalandiya's and indeed can be used to give a simpler proof of Kalandiya's lemma.

For any continuous function $f$ defined on $[-1,1]$, its modulus of continuity is denoted and defined by

$$
\begin{gathered}
w(f ; \delta)=\max _{\left|x_{2}-x_{1}\right| \leq \delta}\left|f\left(x_{2}\right)-f\left(x_{1}\right)\right| . \\
x_{1}, x_{2} \in[-1,1]
\end{gathered}
$$

Two simple properties of $\omega$ which follow immediately from this definition and which we shall use subsequently are

$$
\left\{\begin{array}{l}
\omega(f ; \delta) \leq 2\|f\|_{\infty} \text { and } \\
\omega(f ; \delta) \leq \delta .\left\|f^{\prime}\right\|_{\infty},
\end{array}\right.
$$

the latter result following from the mean value theorem where we assume that $f^{\prime}$ exists and is continuous on $[-1,1]$.

THEOREM 1.2. (Steckin [4]). Suppose $p_{n}$ is a polynomial of degree $\leq$. Then 
(1. 10)

$$
\left|p_{n}^{\prime}(x)\right| \leq\left\{\begin{array}{l}
(n / 2)\left(1-x^{2}\right)^{-\frac{3}{2}} \omega\left(p_{n} ; \pi / n\right),|x|<1, \\
\left(n^{2} / 2\right) \omega\left(p_{n} ; \pi / n\right),|x| \leq 1 .
\end{array}\right.
$$

If we choose $p_{n}=T_{n}$, the Chebyshev polynomial of the first kind, then $\omega\left(T_{n} ; \pi / n\right)=2$. Since $\left|T_{n}^{\prime}(x)\right| \leq n\left(1-x^{2}\right)^{-\frac{3}{2}}$ for $|x|<1$ and since $\left|T_{n}^{\prime}(x)\right| \leq n^{2}$ for $|x| \leq 1$, it follows that the coefficients of $\omega$ on the right hand side of (1.10) cannot be improved.

Returning to Theorem 1.1 (i) we observe that the exponent of $n$ in (1.6) cannot be improved either, in that there exists $f \in \operatorname{Lip} \alpha$ and a polynomial satisfying (1.5) such that $M_{n}(\beta) \geq A_{4} n^{-\alpha+2 \beta}$. To see this suppose we choose $f \equiv 0$ and $p_{n}(x)=A_{1} n^{-\alpha} T_{n}(x)$. Then (1.5) is satisfied. If we choose $x_{1}=1, x_{2}=\cos (\pi / n)$ then, from (1.3),

$$
\begin{aligned}
M_{n}(\beta) & \geq \frac{\left|{ }_{n}\left(x_{2}\right)-{ }_{n}{ }_{n}\left(x_{1}\right)\right|}{\left|x_{2}-x_{1}\right|^{\beta}} \\
& =\frac{A_{1}\left|T_{n}\left(x_{2}\right)-T_{n}\left(x_{1}\right)\right|}{n^{\alpha}\left|x_{2}-x_{1}\right|^{\beta}} \\
& =\frac{2^{1-\beta} A_{1}}{n^{\alpha}(\sin (\pi / 2 n))^{2 \beta}} \\
& \geq \frac{2^{1+\beta} A_{1}}{\pi^{2 \beta}} \cdot n^{-\alpha+2 \beta},
\end{aligned}
$$

which is the desired result.

In this paper we wish to extend the result of Theorem 1.1 to the case when, for some integer $k \geq 1$, the $k$ th order derivative of $f$ is in Lip $\alpha$. We then modify the bound for $\left\|r_{n}\right\|_{\infty}$ as given in (1.5) but again we require bounds for the Lipschitz constant $M_{n}(\beta)$. The results are given in Theorem 2.1. 
2. Functions whose derivatives are Lipschitz continuous.

Without any further ado we shall now state and prove the principal result of this paper.

THEOREM 2.1. Suppose that for some $k \geq 1, f^{(k)} \in$ Lip $\alpha$ and let $p_{n}$ be a polynomial of degree $\leq n$ such that

$$
\left\|r_{n}\right\|_{\infty} \leq A_{5} n^{-k-\alpha}
$$

Then

(i) for every polynomial of degree $\leq n$ satisfying (2.1) and for $O<B \leq 1$

$$
M_{n}(\beta) \leq A_{6} n^{-k-\alpha+2 \beta}
$$

(ii) there exists a polynomial of degree $\leq n$ satisfying (2.1) and such that for $0<\beta \leq 1$,

$$
M_{n}(\beta) \leq A_{7} n^{-k-\alpha+\beta}
$$

Proof. We observe, see Meinardus [3, rheorem 45] that if $p_{n}$ is the polynomial of best uniform approximation to $f$ on $[-1,1]$ then it satisfies (2.1).

(i) First, we shall show that for every polynomial satisfying (2.1),

$$
\left\|r_{n}^{\prime}\right\|_{\infty} \leq A_{8} n^{-k-\alpha+2} \text {. }
$$

Let $q_{n-1}^{*}$ denote the polynomial, of degree $\leq(n-1)$, of best uniform approximation to $f^{\prime}$ on $[-1,1]$. Since the $(k-1)$ st derivative of $f^{\prime}$ is in Lip $\alpha$ we have [3, Theorem 45] that

$$
|| f^{\prime}-q_{n-1}^{*}||_{\infty} \leq A_{g}(n-1)^{-(k-1)-\alpha} \leq A_{10^{n}}{ }^{-k-\alpha+1} \text {, }
$$

for $n \geq 2$. For any $x \in[-1,1]$,

$$
\begin{aligned}
\left|r_{n}^{\prime}(x)\right|= & \left|\left(f^{\prime}(x)-q_{n-1}^{*}(x)\right)+\left(q_{n-1}^{*}(x)-p_{n}^{\prime}(x)\right)\right| \\
& \leq A_{10^{n}}{ }^{-k-\alpha+1}+\left|\left(s_{n}-p_{n}\right)^{\prime}(x)\right|,
\end{aligned}
$$

say, where $s_{n}$ is any polynomial of degree $\leq n$ such that $s_{n}^{\prime}=q_{n-1}^{*}$. By Theorem 1.2, for any $x \in[-1,1]$, 


$$
\begin{aligned}
\left|\left(s_{n}-p_{n}\right)^{\prime}(x)\right| & \leq\left(n^{2} / 2\right) \omega\left(s_{n}-p_{n} ; \pi / n\right) \\
& =\left(n^{2} / 2\right) \omega\left(\left(s_{n}-f\right)+\left(f-p_{n}\right) ; \pi / n\right) \\
& \leq\left(n^{2} / 2\right)\left\{\omega\left(s_{n}-f ; \pi / n\right)+\omega\left(r_{n} ; \pi / n\right)\right\} \\
& \leq\left(n^{2} / 2\right)\left\{(\pi / n)|| s_{n}^{\prime}-f^{\prime}\left\|_{\infty}+2|| r_{n}\right\|_{\infty}\right\},
\end{aligned}
$$

on using (1.9). From (2.1) and (2.5) we find

$$
\left|\left(s_{n}-p_{n}\right)^{\prime}(x)\right| \leq\left\{A_{5}+(\pi / 2) A_{10}\right\} n^{-k-\alpha+2} .
$$

Substituting this back into (2.6) then, since $x$ is arbitrary, we establish (2.4) where $A_{8}=A_{5}+(1+\pi / 2) A_{10}$.

We can now complete the proof of (i). If $x_{1}, x_{2}$ are any two points of $[-1,1]$ such that $\left|x_{2}-x_{1}\right| \geq 1 / n^{2}$ then

$$
\frac{\left|r_{n}\left(x_{2}\right)-r_{n}\left(x_{1}\right)\right|}{\left|x_{2}-x_{1}\right|^{\beta}} \leq 2 A_{5} n^{-k-\alpha+2 \beta} \text {. }
$$

On the other hand if $\left|x_{2}-x_{1}\right| \leq 1 / n^{2}$ then, for $0<\beta \leq 1$, we have

$$
\begin{aligned}
\frac{\left|r_{n}\left(x_{2}\right)-r_{n}\left(x_{1}\right)\right|}{\left|x_{2}-x_{1}\right|^{\beta}} & \leq\left|x_{2}-x_{1}\right|^{1-\beta}|| r_{n}^{\prime}||_{\infty} \\
& \leq A_{8} n^{-k-\alpha+2 \beta},
\end{aligned}
$$

by (2.4). Inequality (2.2) now follows immediately from (2.7) and (2.8) where $A_{6}=\max \left\{2 A_{5}, A_{8}\right\}$.

(ii) To prove (2.3) we shall use an imbedding argument similar to that given by Ioakimidis in [1] for the proof of Theorem 1.1 (ii). First we shall show the existence of a polynomial $p_{n}$ which not only satisfies (2.1) but also

$$
\left\|r_{n}^{\prime}\right\|_{\infty} \leq A_{11} n^{-k-\alpha+1}
$$

To do this, choose $c>1$ and write $x=c t$. On the interval $-1 \leq t \leq 1$, define a function $F(t)$, say, such that (i) $F(k)(t) \in \operatorname{Lip} \alpha$ and $(i i) \quad F(t)=f(c t)$ on $-1 / c \leq t \leq 1 / c$. In the intervals $[-1,-1 / c]$ 
and $[1 / c, 1]$ we need to continue the definition of $f$ so that $F$ possesses a continuous $k$ th order derivative which, on $-1 \leq t \leq 1$, is in Lip $\alpha$. One way of doing this is to define $F^{(k)}$ as

$$
F^{(k)}(t)= \begin{cases}f^{(k)}(-1) & ,-1 \leq t \leq-1 / c, \\ f^{(k)}(c t) & ,-1 / c \leq t \leq 1 / c, \\ f^{(k)}(1), & 1 / c \leq t \leq 1,\end{cases}
$$

and then to recover $F$ by integration choosing constants of integration appropriately on $[-1,-1 / c]$ and $[1 / c, 1]$. Let $P_{n}^{*}$ denote the polynomial of best uniform approximation of degree $\leq n$ to $F$ on $-1 \leq t \leq 1$. Then, since $F^{(k)} \in \operatorname{Lip} \alpha,\left\|\mid F-P_{n}^{*}\right\|_{\infty} \leq A_{12} n^{-k-\alpha}$. For $-1 \leq x \leq 1$, choose

$$
p_{n}(x)=p_{n}(c t)=P_{n}^{*}(t),-1 / c \leq t \leq 1 / c .
$$

Then certainly (2.1) is satisfied for this polynomial. Now, for $-1 \leq x \leq 1$,

$$
\begin{aligned}
r_{n}^{\prime}(x) & =\frac{1}{c} \frac{d}{d t}\left(F(t)-P_{n}^{*}(t)\right) \\
& =\frac{1}{c}\left\{\left(\frac{d F}{d t}-Q_{n-1}^{*}(t)\right)+\left(Q_{n-1}^{*}(t)-\frac{d P_{n}^{*}}{d t}\right)\right\},
\end{aligned}
$$

where $Q_{n-1}^{*}(t)$ is the polynomial of best uniform approximation of degree $\leq(n-1)$ to $d F / d t$ on $-1 \leq t \leq 1$, Again, for $n \geq 2$ and any $t \in[-1,1]$ we know that

$$
\left|\frac{d F}{d t}-Q_{n-1}^{*}(t)\right| \leq A_{13^{n}}{ }^{-k-\alpha+1} .
$$

If we define $S_{n}(t)$ to be a polynomial of degree $\leq n$ such that $d S_{n} / d t=Q_{n-1}^{*}(t)$ then from $(2.10)$ and (2.11) we have

$$
c\left|p_{n}^{\prime}(x)\right| \leq A_{13^{n}}{ }^{-k-\alpha+1}+\left|\frac{d}{d t}\left(S_{n}(t)-P_{n}^{*}(t)\right)\right|
$$

For points in $[-1 / c, 1 / c]$ we have, by Theorem 1.2 , 


$$
\begin{aligned}
\left|\frac{d}{d t}\left(S_{n}(t)-P_{n}^{*}(t)\right)\right| \leq \frac{n}{2\left(1-t^{2}\right)^{\frac{\pi}{2}}} \omega\left(S_{n}-P_{n}^{*} ; \frac{\pi}{n}\right) \\
\leq \frac{n c}{2\left(c^{2}-1\right)^{\frac{3}{2}}}\left\{\omega\left(S_{n}-F ; \frac{\pi}{n}\right)+\omega\left(F-P_{n}^{*} ; \frac{\pi}{n}\right)\right\} \\
\leq \frac{n c}{2\left(c^{2}-1\right)^{\frac{7}{2}}}\left\{\frac{\pi}{n} \max _{-1 \leq t \leq 1}\left|\frac{d F}{d t}-Q_{n-1}^{*}(t)\right|+\right. \\
\left.\quad+2 A_{12^{n}}{ }^{-k-\alpha}\right\} \\
\leq \frac{c}{2\left(c^{2}-1\right)^{\frac{3}{2}}}\left(\pi A_{13}+2 A_{12}\right)^{-k-\alpha+1},
\end{aligned}
$$

on using (2.11). Combining (2.13) with (2.12) gives (2.9) where $A_{11}=A_{12}\left(c^{2}-1\right)^{-\frac{1}{2}}+A_{13}\left\{(\pi / 2)\left(c^{2}-1\right)^{-\frac{1}{2}}+c^{-1}\right\}$.

We can now complete the proof of (ii). If $x_{1}, x_{2}$ are any two points of $[-1,1]$ such that $\left|x_{2}-x_{1}\right| \geq 1 / n$ then

$$
\frac{\left|r_{n}\left(x_{2}\right)-r_{n}\left(x_{1}\right)\right|}{\left|x_{2}-x_{1}\right|^{\beta}} \leq 2 A_{5} n^{-k-\alpha+\beta} .
$$

On the other hand if $\left|x_{2}-x_{1}\right| \leq 1 / n$ then, from (2.9),

$$
\begin{aligned}
\frac{\left|r_{n}\left(x_{2}\right)-r_{n}\left(x_{1}\right)\right|}{\left|x_{2}-x_{1}\right|^{\beta}} & \leq\left|x_{2}-x_{1}\right|^{1-\beta} \cdot|| r_{n}^{\prime}||_{\infty} \\
& \leq A_{11} n^{-k-\alpha+\beta},
\end{aligned}
$$

provided that $B \leq 1$. From (2.14) and (2.15), (2.3) follows where we choose $A_{7}=\max \left\{2 A_{5}, A_{11}\right\}$. This completes the proof of the theorem. As a final comment we note, by choosing $f \equiv 0, p_{n}=A_{5} n^{-k-\alpha_{T}} T_{n}$ and taking points $x_{1}=1, x_{2}=\cos (\pi / n)$, that the exponent of $n$ in (2.2) cannot be improved.

\section{References}

[1] N.I. Ioakimidis, "An improvement of Kalandiya's theorem", J. Approx. Theory 38 (1983), 354-356. 
[2] A.I. Kalandiya, "On a direct method of solution of an equation in wing theory and its application to the theory of elasticity", Mat. Sb. 42 (1957), 249-272. (In Russian)

[3] G. Meinardus, Approximation of functions: theory and numerical methods, (Springer-Verlag, Berlin, Heidelberg, New York, 1967).

[4] S.B. Steckin, "Generalization of some Bernstein inequalities", Dok 2. Akad. Nauk SSSR 60 (1948), 1511-1514. (In Russian).

Department of Mathematics,

University of Tasmania,

Box 252C, G.P.O.,

Hobart,

Tasmania,

Australia. 